\title{
Functional insights into pGI2, a cryptic rolling-circle replicating plasmid from Bacillus thuringiensis
}

\author{
Lieve Hoflack, ${ }^{1}+$ Andrea Wilcks, ${ }^{2}$ Lars Andrup ${ }^{2}$ and Jacques Mahillon ${ }^{3}$
}

Author for correspondence: Jacques Mahillon. Tel: +3210473370 . Fax: +3210473440 .

e-mail:mahillon@mbla.ucl.ac.be

1 Laboratorium Genetica, Universiteit Gent, K. L. Ledeganckstraat 35, and Plant Genetic Systems, J. Plateaustraat 22, B-9000 Gent, Belgium

2 National Institute of Occupational Health, Lerse Parkallé 105, DK-2100 Copenhagen, Denmark

3 Laboratoire de Génétique Microbienne, Université catholique de Louvain, Place Croix du Sud, 2/12, B-1348 Louvain-la-Neuve, Belgium

\begin{abstract}
Detailed functional analysis revealed the modular organization of pGI2, a 9672 bp plasmid from Bacillus thuringiensis H1.1 that harbours the 4149 bp transposon Tn4430. Whereas the pGI2 leading-strand replicon was identified through deletion experiments, sequence comparisons indicated the presence of an SsO $_{\mathrm{t}}$-like single-strand origin commonly found among Bacillus plasmids. Southern hybridization confirmed the existence of SsDNA intermediates, but only in the case of plasmid derivatives lacking the sso, site. Moreover, the pGI2 replication protein Rep displayed significant similarity with that of pTX14-3, a $7.6 \mathrm{~kb}$ plasmid from B. thuringiensis serovar israelensis, suggesting that both elements are representatives of a new family of rolling-circle replicating (RCR) plasmids. In addition, both plasmids share a conserved 320 bp region downstream of their rep genes which, in the case of pG12, appeared indispensable for replication. This region is therefore likely to correspond to, or to be part of, the actual double-strand origin of both plasmids. Another interesting feature of pG12 is the presence of a mobilization (Mob) protein, as demonstrated by its ability to be mobilized by the conjugative plasmid pAW63 from B. thuringiensis serovar kurstaki HD73. The same transfer system was also used to unambiguously demonstrate similar properties of the related Mob-like protein from pTX14-3. A closer analysis of this family of related Mob proteins suggested a subdomanial organization among its members. Finally, the 270 residue PGI2 ORF2 was shown to be related to ORF43 of pMRC01, a $60 \mathrm{~kb}$ conjugative plasmid from Lactococcus lactis subsp. lactis. Although no function has been assigned to the putative ORF43 protein, it is located downstream of a bacteriocin operon, next to an IS946 element. pGI2 appears thus far as an assemblage of functional modules with no obvious metabolic function, presumably acting as a reservoir of carrier (rep and sso), rearrangement (Tn4430) or recruiting (Mob) entities for its bacterial host.
\end{abstract}

Keywords: Bacillus thuringiensis, mobilization, plasmid, rolling circle replication, ssDNA

\section{INTRODUCTION}

One of the major characteristics of rolling-circle replicating (RCR) plasmids (reviewed by Khan, 1997) is their modular construction. Even the replication of the leading and lagging strands is governed by distinct modules. The minimal replicon responsible for leading

† Present address: Department of Pediatric Dentistry, University of Texas, 7703 Floyd Curl Drive, San Antonio, TX 78284, USA

Abbreviations: IR, inverted repeat; RCR, rolling-circle replicating. strand replication consists of the gene encoding the initiator Rep protein and the origin where leading strand replication begins, called the double-strand origin (dso). Based on sequence similarities within this minimal replicon module, RCR plasmids have been divided into several families with pT181 (reviewed by Wang et al., 1993), pC194 (reviewed by Seery et al., 1993; NoirotGros et al., 1994), pE194 (Novick, 1989), pSN2 (Khan \& Novick, 1982), pTX14-3 (Jannière et al., 1993; Andrup et al., 1994) and pGI3 (Hoflack et al., 1997) as representatives. 
Lagging strand replication mainly relies on host-encoded functions and starts from the so-called single-strand origin (sso), which is the sole element comprising the lagging strand replication module. It is located in a noncoding region with high potential to form imperfect stem-loop structures. However, since sso activity is orientation-dependent, it does not rely entirely on secondary structures, but also on the DNA sequences themselves (del Solar et al., 1993; Seery \& Devine, 1993). Although there is generally a good correlation between the sso type and plasmid host range, each particular sso being active only in closely related bacteria, some exceptions do exist. The $s o_{\mathrm{t}}$ type found in native Bacillus plasmids and first reported in pBAA1 (Devine $e t$ al., 1989) was also shown to be active in Staphylococcus aureus (Seery \& Devine, 1993). Similarly, the sso $_{\mathrm{u}}$ type, originally isolated from Staphylococcus, is functional in multiple Gram-positive bacteria, including Bacillus strains (Boe et al., 1989).

Another module often present in Gram-positive RCR plasmids is a mobilization entity that enables plasmid transmission between bacteria during conjugation (Guzmán \& Espinosa, 1997; Meijer et al., 1998). As pointed out by Ilyina \& Koonin (1992), the organization and activity (DNA nicking and strand displacement) of the plasmid-transfer module are reminiscent of those of the minimal replicon, with the mobilization protein (Mob) and origin of transfer (oriT) corresponding to the Rep and $d s o$, respectively. This mobilization module is however not restricted to RCR elements, but is also present in $\theta$-replicating plasmids. Indeed, detailed comparisons of oriT sites and Mob proteins have so far revealed four families: three from $\theta$-replicating plasmids (Lanka \& Wilkins, 1995) and that from the Grampositive RCR plasmids (Guzmán \& Espinosa, 1997; Meijer et al., 1998; Ilyina \& Koonin, 1992).

Other modules may be present as well, but far less is known about their potential biological role. For the Bacillus subtilis plasmids pTA1015, pTA1040, pTA1050 and pTA1060, several putative metabolic modules have been identified through their similarity to existing chromosomal systems. However, experimental confirmation of their function is still required (Meijer $e t a l$., 1998). In Bacillus thuringiensis, even less is known about the loci present on cryptic plasmids. In the presumably $\theta$-replicating plasmid $\mathrm{pHT} 1030$, the only features identified so far are the segregational stability gene $s p b A$ (Lereclus \& Arantes, 1992) and the mobile elements IS231 and Tn4430, both often found in the $B$. thuringiensis genome (Mahillon et al., 1994; Léonard et al., 1997).

pGI2 is the second smallest plasmid from $B$. thuringiensis $\mathrm{H} 1.1$, a strain containing at least six plasmids: three large ones $(>50 \mathrm{~kb})$ and three small ones $(<15 \mathrm{~kb}$ ) called pGI1, pGI2 and pGI3 (Mahillon et al., 1988). Genetic characterization of pGI3, the largest of the three small molecules, revealed that it is a RCR plasmid and defines, together with pSTK1 from Bacillus stearothermophilus, a new family of rolling-circle replicons (Hoflack et al., 1997). pGI1, the smallest one, is also a RCR plasmid belonging to the pC194 family ( $\mathrm{L}$. Hoflack \& J. Mahillon, unpublished results). pGI2 was shown to be a 9672 bp molecule containing the transposon Tn4430 (Mahillon \& Seurinck, 1988). In this paper, we report on the detailed functional analysis of pGI2, including its double-strand and single-strand replication module, the activity of its Mob proteins and the influence of different modules on its segregational stability. The distribution of the pGI2 replicon among members of the Bacillus cereus group is also analysed.

\section{METHODS}

Bacterial strains and plasmids. Tables 1 and 2 list the bacterial strains and plasmids used in this study. Media and growth conditions were as previously described (Hoflack et al., 1997). When required, antibiotics were added at the following concentrations: $100 \mu \mathrm{g}$ ampicillin $\mathrm{ml}^{-1}, 4 \mu \mathrm{g}$ tetracycline $\mathrm{ml}^{-1}$, $6 \mu \mathrm{g}$ chloramphenicol $\mathrm{ml}^{-1}, 20 \mu \mathrm{g}$ nalidixic acid $\mathrm{ml}^{-1}, 100 \mu \mathrm{g}$ streptomycin $\mathrm{ml}^{-1}, 20 \mu \mathrm{g}$ erythromycin $\mathrm{ml}^{-1}$ for Bacillus and $200 \mu \mathrm{g}$ erythromycin $\mathrm{ml}^{-1}$ for Escherichia coli. All antibiotics were obtained from Sigma, except for erythromycin (from Duchefa).

DNA manipulations. Restriction enzymes were purchased from New England Biolabs, Klenow enzyme and T4 DNA ligase from Boehringer Mannheim and Taq polymerase from Pharmacia Biotech. All enzymes were used as specified by the manufacturers. DNA fragments were isolated from agarose gels using Gene Clean II (Bio101). Plasmid DNA isolations were performed as described by Voskuil \& Chambliss (1993) for Bacillus and as described by Sambrook et al. (1989) for E. coli. Total DNA from Bacillus was prepared as previously reported (Hoflack et al., 1997). Sequencing was performed with the AutoRead 1000 Sequencing Kit and the Automated Laser Fluorescent ALF DNA sequencer of Pharmacia Biotech. The sequencing primers were designed with the oligo program (version 4.0, National Biosciences, Plymouth, MI, USA). DNA and protein analyses were performed with the IntelliGenetics software, release 5.4 (IntelliGenetics, Mountain View, CA, USA). Database searches were performed with the GCG package (Genetics Computer Group, version 8, Madison, WI, USA). The 530 bp pGI2 fragment used in the distribution study was obtained by PCR with primers $5^{\prime}$ GAGAAAAAAGAAAAGT $-3^{\prime}$ and $5^{\prime}$-TAATAAATAATGCGTATGTA-3', amplifying a DNA region corresponding to positions $10-168$ of the 204 residue Rep protein. The conditions used were those described for the detection of pGI3 (Hoflack et. al., 1997).

Plasmid constructions. Table 2 gives the description of the constructions used in this study. pGI2 was cloned into pGI401 (Josson et al., 1989) as a Sall fragment, resulting in pGI208. Self-ligation of the 9009 bp KpnI fragment generated pGI2203, a pGI2 derivative lacking Tn4430. Because pGI2203 contains two BanII sites, pGI2205 was constructed by ligating the 6675 bp PacI-Sall pGI2203 fragment and the 1956 bp BanIISalI pGI2203 fragment. Further details of the constructs described in Table 2 are available on request.

Mating in broth. Mobilization of the different pGI2 derivatives was done as previously described (Andrup et al., 1996). The donor strain AW17, a B. thuringiensis serovar kurstaki HD73 strain cured of pHT73, one of its two conjugative plasmids, was resistant to streptomycin. Selection of the transconjugants was performed on erythromycin and nalidixic acid, to which the recipient strain (AW43, B. thuringiensis kurstaki HD73 cured of both conjugative plasmids) was resistant. The 
Table 1. Bacterial strains used in this study

\begin{tabular}{|c|c|c|}
\hline Strain & Description & Reference or source \\
\hline \multicolumn{3}{|l|}{ E. coli } \\
\hline MC1061 & $\begin{array}{l}\mathrm{F}^{-} \text {araD139 } \Delta(\text { ara-leu }) 7696 \text { galE15 galK16 } \\
\Delta(\text { lac }) X 74 \operatorname{rpsL}\left(\mathrm{Str}^{\mathrm{R}}\right) \text { hsdR2 }\left(\mathrm{r}_{\mathbf{K}}^{-} \mathrm{m}_{\mathbf{K}}^{+}\right) \text {mcrA } \\
m c r B 1\end{array}$ & Wertman et al. (1986) \\
\hline GM2163 & $\begin{array}{l}\mathrm{F}^{-} \text {ara-14 leuB6 thi-1 fbuA31 lacY1 ts } x-78 \text { galK2 } \\
\text { galT22 supE44 bisG4 rpsL136 }\left(\mathrm{Str}^{\mathrm{R}}\right) \text { xyl-5 } \\
\text { mtl-1 dam13:: Tn9 }\left(\mathrm{Cm}^{\mathrm{R}}\right) \text { dcm-6 mcrB1 bsdR2 } \\
\left(\mathrm{r}_{\mathrm{K}}^{-} \mathrm{m}_{\mathrm{K}}^{+}\right) \text {mcrA }\end{array}$ & Woodcock et al. (1989) \\
\hline \multicolumn{3}{|l|}{ B. subtilis } \\
\hline CU267 & ilvB2 leuB16 trpC2, Str ${ }^{\mathrm{R}}$ & Jones \& Errington (1987) \\
\hline \multicolumn{3}{|l|}{ B. thuringiensis } \\
\hline AW 17 & HD73 cured of pHT73, $\mathrm{Str}^{\mathrm{R}}$ & Wilcks et al. (1998) \\
\hline AW43 & HD73 cured of both pAW63 and pHT73, $\mathrm{Nal}^{\mathrm{R}}$ & Wilcks et al. (1998) \\
\hline AW48 & 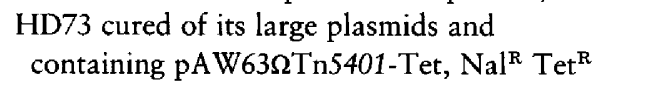 & Wilcks et al. (1998) \\
\hline GBJ001 & israelensis strain, $\mathrm{Str}^{\mathrm{R}}$ & Jensen et al. (1995) \\
\hline GBJ002 & israelensis strain, $\mathrm{Nal}^{\mathrm{R}}$ & Jensen et al. (1996) \\
\hline HD73 & $\begin{array}{l}\text { kurstaki strain devoid of pGI2-related } \\
\text { sequences }\end{array}$ & $\mathrm{IEBC}^{*}$ \\
\hline Dipel & Important commercial strain & Abbott Laboratories, IL, USA \\
\hline $\begin{array}{l}\text { Berliner 1715, Bt1, H1.1, HD14, T01 002, T01 } \\
\text { 027, T02 001, T03A001, T03A016, T03 001, } \\
\text { T03B001, T03C001, T04B001, T05 001, } \\
\text { T05A013, T06A001, T07 029, T08 018, } \\
\text { T08B001, T09 001, T10 001, T11 001, T12 } \\
\text { 001, H14, T16 001, T18 001, T20 001, T21 } \\
\text { 001, T24 001, T26 001, T28A001, T30 001, } \\
\text { T42 001, T45 } 001\end{array}$ & $\begin{array}{l}\text { Strains tested for the presence of the pGI2 } \\
\text { replicon }\end{array}$ & $\mathrm{IEBC}^{*}$ \\
\hline B. cereus & $\begin{array}{l}\text { Strains tested for the presence of the pGI2 } \\
\text { replicon }\end{array}$ & \\
\hline $\begin{array}{l}\text { ATCC } 10876, \text { ATCC } 10987, \text { ATCC } 14579, \\
\text { ATCC } 21281, \text { ATCC } 21282\end{array}$ & & Carlson et al. (1994) \\
\hline CER001, CER002, CER052, CER116, CER484 & & IEBC $^{*}$ \\
\hline B. mycoides & $\begin{array}{l}\text { Strains tested for the presence of the pGI2 } \\
\text { replicon }\end{array}$ & \\
\hline $\begin{array}{l}\text { KBS1-4, KBS1-5, KBS3-1, KNC1-10, KNC2-18, } \\
\text { KNC3-15 }\end{array}$ & & Bell \& Friedman (1994) \\
\hline $\mathrm{MYC003}$ & & $\mathrm{IEBC}^{*}$ \\
\hline $\begin{array}{l}\text { NRRL B-3436, NRRL BD-3, NRRL NRS1216, } \\
\text { NRRL NRS306, NRRL NRS321, NRRL } \\
\text { NRS323 }\end{array}$ & & Nakamura \& Jackson (1995) \\
\hline
\end{tabular}

*IEBC, International Entomopathogenic Bacillus Center, Pasteur Institute, Paris, France

experiments were performed at least twice, except for pGI2204-49, which was only tested once.

For pTX14-3 mobilization, the conjugation experiments were conducted in a plasmid-cured strain of $B$. thuringiensis serovar israelensis. Two $\mathrm{Cm}^{\mathrm{R}}$ derivatives of $\mathrm{pTX14-3}$, one containing the intact mob gene (pAND007) and the other containing a deletion-derivative (pAND008) (Andrup et al., 1995), were electroporated into the streptomycin-resistant $B$. thuringiensis serovar israelensis strain GBJ001 (Jensen et al., 1995). An isolate of each transformant was verified by restriction analysis and used as recipient in mating with a AW48 donor containing pAW63:: Tn5401( Tet $^{\mathrm{R}}$ ) (Wilcks et al., 1998). One of each transconjugant containing both pAW63:: Tn5401 and either pAND007 or pAND008 were in turn used as donors in mating experiments with a $\mathrm{Nal}^{\mathrm{R}}$ recipient (GBJ002, Jensen et al., 1996). The transfer frequencies of both the conjugative plasmid pAW63 and the pTX14-3 derivatives were determined in short-term mating experiments (mean of three experiments).

Assay for segregational stability. The different plasmids tested for their segregational stability were electroporated into strain AW17 (a Str ${ }^{\mathrm{R}}$ derivative of HD73) and the recombinant clones were grown at $30^{\circ} \mathrm{C}$ without antibiotic selection. After $10,20,30$ and 40 generations, aliquots were spread on 
Table 2. Plasmids used in this study

\begin{tabular}{|c|c|c|}
\hline Plasmid & Description & Reference or source \\
\hline pAND007 & $\begin{array}{l}\text { A pTX14-3 derivative containing an intact mob gene, } \\
\mathrm{Cm}^{\mathrm{R}}\end{array}$ & Andrup et al. (1995) \\
\hline pAND008 & $\begin{array}{l}\text { A pTX14-3 derivative containing a deleted version of } \\
\text { the } m o b \text { gene, } \mathrm{Cm}^{\mathrm{R}}\end{array}$ & Andrup et al. (1995) \\
\hline pGI401 & pBR322 carrying the Ery ${ }^{\mathrm{R}}$ gene from $\mathrm{pAM} \beta 1$ & Josson et al. (1989) \\
\hline pGI2 & $\begin{array}{l}9672 \text { bp cryptic plasmid from } B \text {. thuringiensis strain } \\
\text { H1.1 }\end{array}$ & $\begin{array}{l}\text { Mahillon \& Seurinck } \\
\text { (1988) }\end{array}$ \\
\hline pGI208 & pGI2 cloned into pGI401 as a Sall fragment & This study \\
\hline pGI2203 & $\begin{array}{l}\text { Largest KpnI fragment of pGI208; pGI208 without } \\
\text { Tn } 4430\end{array}$ & This study \\
\hline pGI2204 & $\begin{array}{l}\text { Largest } P m l \mathrm{I} \text { fragment of pGI2203; pGI2203 with sso } \\
\text { and } m o b \text { partially deleted }\end{array}$ & This study \\
\hline pGI2205 & $\begin{array}{l}381 \text { bp PacI-BanII deletion of pGI2203; pGI2203 } \\
\text { without sso }\end{array}$ & This study \\
\hline pGI2209 & $\begin{array}{l}422 \text { bp PacI-BstXI deletion of pGI2203; pGI2203 } \\
\text { without sso and oriT }\end{array}$ & This study \\
\hline pGI2210 & $\begin{array}{l}2337 \text { bp PacI-SalI deletion of pGI2203; pGI2203 } \\
\text { without mob, sso and oriT }\end{array}$ & This study \\
\hline pGI2211 & $\begin{array}{l}1369 \text { bp EcoRV-SalI deletion of pGI2203; pGI2203 } \\
\text { without mob }\end{array}$ & This study \\
\hline pGI2204b & $\begin{array}{l}2652 \text { bp NheI-SalI deletion of pGI2204; pGI2204 } \\
\text { without ORF2 and ORF3 }\end{array}$ & This study \\
\hline pGI2204c & $\begin{array}{l}1331 \text { bp BstEII-PacI deletion of pGI2204; pGI2204 } \\
\text { without ORF2 and ORF3 }\end{array}$ & This study \\
\hline pGI2204d & $\begin{array}{l}1551 \text { bp NheI-PacI deletion of pGI2204; pGI2204 } \\
\text { without ORF2 and ORF3 }\end{array}$ & This study \\
\hline pGI2204-61 & $\begin{array}{l}\text { pGI2204 with the rep gene inactivated by a } 330 \mathrm{bp} \\
\text { Nsil-BstBI deletion }\end{array}$ & This study \\
\hline pGI2204-53 & $\begin{array}{l}\text { pGI2204 with ORF2 missing a } 485 \text { bp BstEII-MscI } \\
\text { N-terminal fragment }\end{array}$ & This study \\
\hline pGI2204-49 & $\begin{array}{l}\text { pGI2204 without ORF2 and ORF3, deleted by a } \\
846 \text { bp Mscl-Pacl restriction }\end{array}$ & This study \\
\hline
\end{tabular}

streptomycin-containing plates. Plasmid stability was estimated by calculation of the $\mathrm{Ery}^{\mathrm{R}} / \mathrm{Str}^{\mathrm{R}}$ ratio obtained with 200 individual c.f.u. The experiment was performed twice.

ssDNA detection. ssDNA detection experiments were performed with total DNA from B. thuringiensis $\mathrm{H} 1.1$ and $B$. thuringiensis HD73 transformed with pGI2204c and pGI2210. Total DNA was prepared as previously described (Hoflack et al., 1997) from exponentially growing cultures that were incubated with $100 \mu \mathrm{g}$ rifampicin $\mathrm{ml}^{-\mathbf{1}}$ for $10 \mathrm{~min}$ prior to harvesting. ssDNA detection (Noirot-Gros \& Ehrlich, 1994) was done with or without $\$ 1$ nuclease treatment of the DNA samples, and with or without DNA denaturation before blotting. Southern analysis was performed with a $1137 \mathrm{bp}$ DIG-labelled probe (Boehringer) corresponding to a KpnINhel pGI2 fragment harbouring the rep gene.

\section{RESULTS}

\section{Updating pGI2 sequence and organization}

Database searches with the predicted products of the cryptic ORFs from pGI2 (ORF1, ORF2 and ORF3) revealed that ORF1 showed similarity with the Cterminal part of the Rep protein of pTX14-3, a $B$. thuringiensis israelensis plasmid (Andrup et al., 1994). However, the N-terminal part of the pTX14-3 Rep protein also displayed similarity to a short ORF upstream of ORF1. This prompted us to revise the pGI2 sequence (EMBL accession no. X13481): three A/T bp were deleted, at positions 5031, 5079 and 6640, and the $\mathrm{G} / \mathrm{C}$ bp at position 6732 was replaced by an $A / T$ bp. These revisions extended ORF2 by 67 aa at the Cterminal end (now 270 aa) and ORF1 by 89 aa at its Nterminal end (now $204 \mathrm{aa}$ ).

Surprisingly, comparison with the databases also indicated that the 270 residue ORF 2 displayed $39 \%$ identity ( $52 \%$ for the C-terminal half) with the 286 aa ORF43 of pMRC01 from Lactococcus lactis subsp. lactis (data not shown). This $60 \mathrm{~kb}$ conjugative plasmid is a bacteriocinencoding element whose complete sequence was recently determined by Dougherty et al. (1998). However, although ORF43 is located immediately downstream of the bacteriocin operon, there was no indication of its possible involvement in bacteriocin production or immunity. The putative function of pGI2 ORF2 and ORF3 thus far remains cryptic. 


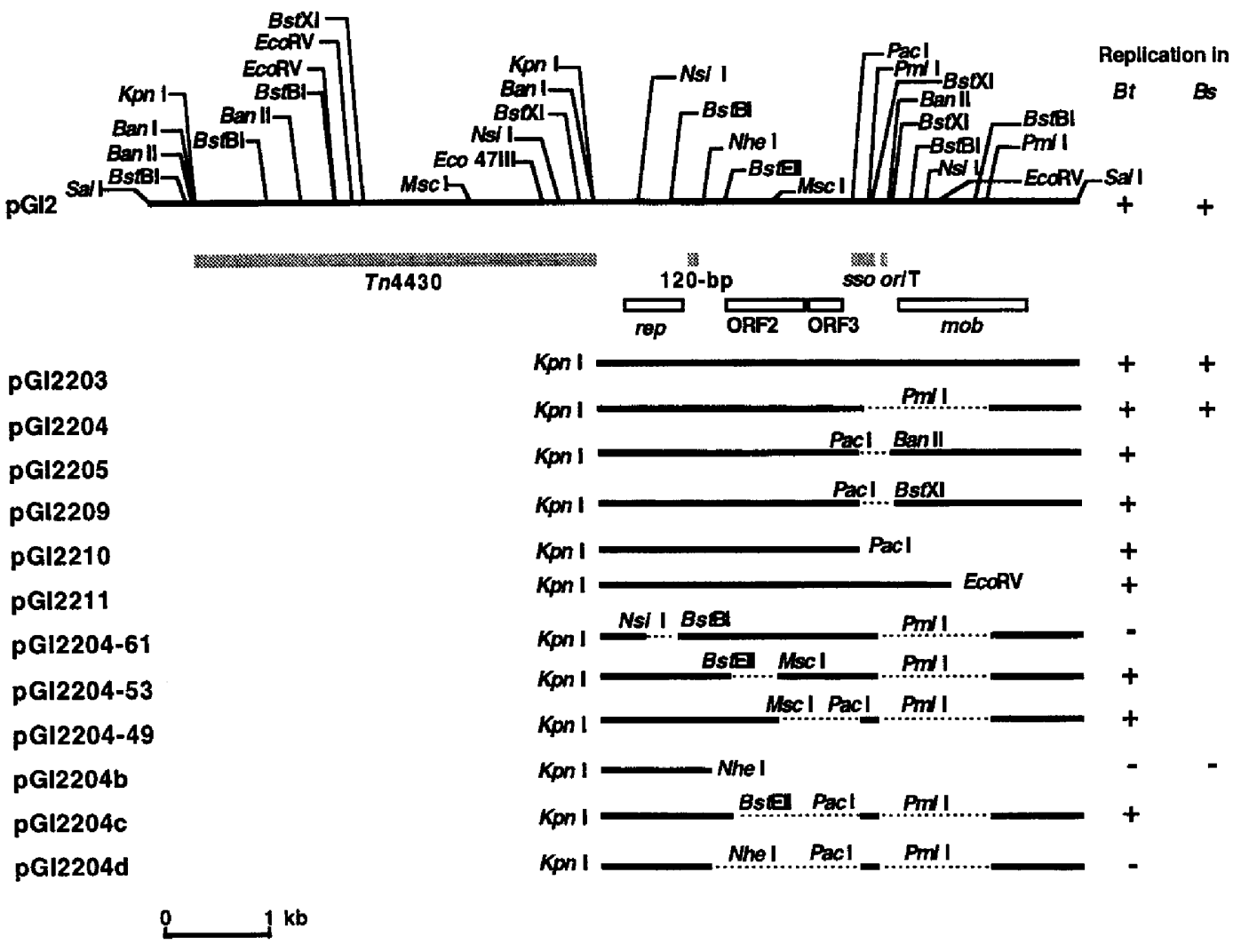

Fig. 1. Structural organization of pGI2 and determination of its minimal replicon. ORFs are indicated as white boxes, with the frames reading from left to right. Tn4430, the 120 bp region homologous to PGI3 and pTX14-3, the sso and the oriT site are indicated as grey boxes. Ability $(+)$ or inability $(-)$ of the different constructs to replicate in $B$. thuringiensis (Bt) and $B$. subtilis $(B s)$ is indicated at the end of each plasmid. Restriction enzymes used for the different constructs are indicated. Note that the cleavage site of $K p n l$ is also present in the vector.

\section{Leading strand replication}

The revised ORF1 (Fig. 1, coordinates 4859-5473) sequence showed $30 \%$ identity ( $46 \%$ similarity) to the pTX14-3 Rep protein (Fig. 2a), indicating that it may represent the pGI2 rep gene. Stepwise deletion derivatives of pGI2 were made in E. coli (Fig. 1 and Table 2) and subsequently transferred to $B$. thuringiensis and/or $B$. subtilis to test their replication capacity. The transformation efficiency of the Bacillus cells ranged from $10^{4}$ to $10^{5}$ c.f.u. ( $\left.\mu \mathrm{g} \mathrm{DNA}\right)^{-1}$. As shown in Fig. 1 , the minimal replicon was determined to be a 1358 bp KpnI-BstEII fragment (pGI2204c) containing only ORF1 as potential coding sequence. Deletion of a 325 bp Nsil-Bst BI fragment internal to ORF1 (Fig. 1, pGI2204-61) abolished its replication capacity, strongly arguing for ORF1 being the pGI2 rep gene.

As indicated below, Southern hybridization revealed that pGI2 is a RCR plasmid. A search for GC-rich stem-loop structures, iterons or sequences that were also present in PTX14-3 did not show any potential $d s o$ upstream of or inside their rep genes. Interestingly however, the presence of a $220 \mathrm{bp}$ NheI-BstEII fragment located $310 \mathrm{bp}$ downstream of the rep gene also appeared to be indispensable for pGI2 replication, since we were unable to transform $B$. thuringiensis with either
pGI2204b or pGI2204d (Fig. 1). Detailed comparison of this pGI2 region with the entire pTX14-3 sequence revealed that, in both plasmids, a $320 \mathrm{bp}$ segment downstream of their rep genes was $84 \%$ identical (compared to the 59\% identity of their rep genes) and contained a 106 bp GC-rich region (coordinates 56985803 and 1765-1870, for pGI2 and pTX14-3, respectively) showing $98 \%$ identity (Fig. 2b). In pGI2, this extremely conserved sequence overlaps with the $220 \mathrm{bp}$ NheI-BstEII segment (positions 5780-5999) required for replication (Figs 1 and $2 \mathrm{~b}$ ). Therefore, based on these observations, it is tempting to suggest that these regions located downstream of the rep gene may correspond to pGI2 and pTX14-3 dso, a structural organization not reported previously.

Some RCR plasmids [at least some of the $\mathrm{pE} 194$ family members (Espinosa et al., 1995) and pSTK1 from the pGI3 family (Narumi et al., 1995)] have been shown to be active in both Gram-positive and Gram-negative hosts. In order to test this possibility for $\mathrm{pGI} 2$, a derivative harbouring its replicon fused to the pAM $\beta 1$ erythromycin resistance gene was constructed in $B$. subtilis (pGI2e, data not shown) and subsequently transformed into E. coli MC1061. Although the transformation efficiency of these $E$. coli cells ranged from $10^{8}$ 
(a)

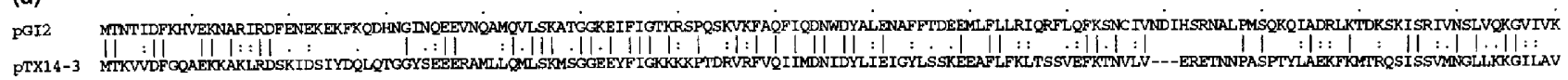

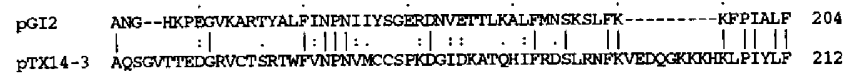

(b)

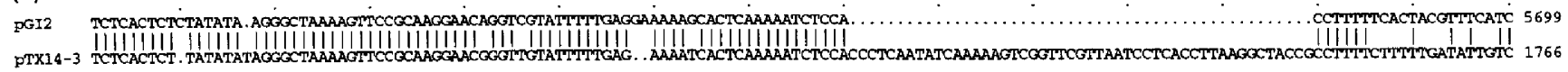
mor

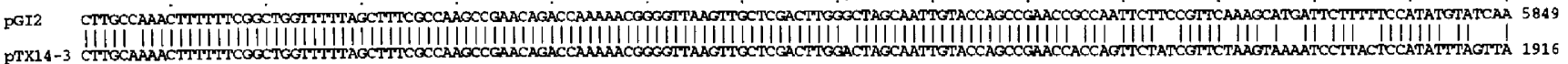

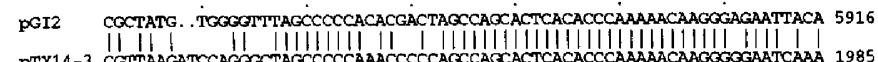

Fig. 2. Comparison of the Rep proteins and putative dso from pGI2 and pTX14-3. (a) Protein alignment was performed with the GAP program in the GCG software package. Identical residues are indicated by vertical bars, conservative substitutions by colons, distantly related residues by dots and gaps by dashes. The Rep sizes are indicated at the end of the proteins. (b) Comparison of DNA sequences downstream of the pGI2 (coordinates 5600-5916) and pTX14-3 (coordinates 1620-1985) rep genes. Identical bp are indicated by vertical bars and gaps (dots) have been introduced to optimize the alignment. The position of the Nhel restriction site is also indicated at positions 5780-5785.
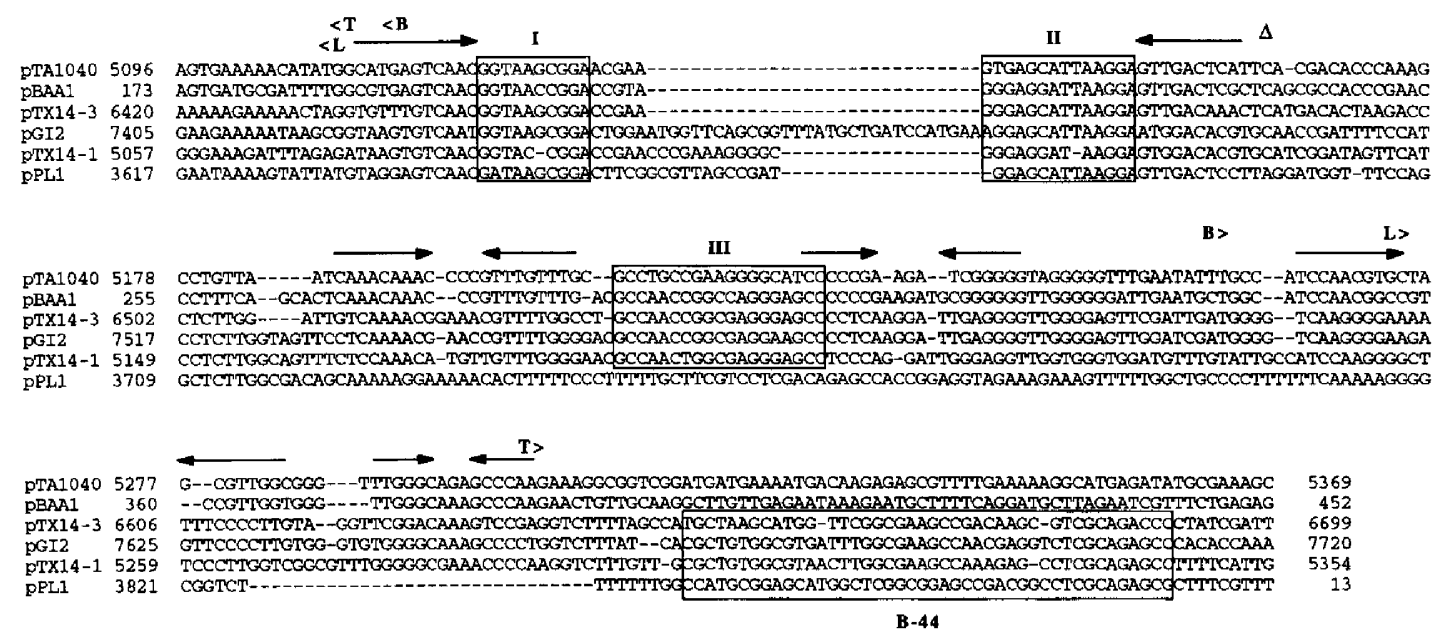

Fig. 3. Alignment between the sso-like sequence of pGI2 and the sso of pTA1040 and pBAA1 from $B$. subtilis, pTX14-3 and pTX14-1 from $B$. thuringiensis and pPL1 from $M$. halophilus. pBAA1 is an $\mathrm{ss}_{\mathrm{t} 1}$-type origin and pTA1040 an $\mathrm{ssO}_{\mathrm{t} 2}$-type. Putative stem-loop structures are indicated as horizontal arrows above the sequences (Meijer et al., 1995). The conserved motifs (Seery \& Devine, 1993) are shown as boxes I-III, whereas the boxed sequences labelled B-44 represent the conserved 44 bp region present in pG12, pTX14-1, pTX14-3 and pPL1. Note that the putative pPL1 sso only harbours the first two conserved boxes and IR. The minimal region for efficient sso activity, as experimentally determined, is shown as $<B$ and $B>$ for $p B A A 1,<L$ and $L>$ for $\mathrm{pLS1}\left(\mathrm{ssO}_{\mathrm{t} 1}\right.$-type), and $<T$ and $T>$ for $\mathrm{pTA1060}$. The remaining part of the sso still present in pGI2204-49 and pGI2204c is located upstream of ' $\Delta$ '. Coordinates of the sequences are indicated.

to $10^{9}$ c.f.u. ( $\left.\mu \mathrm{g} \mathrm{DNA}\right)^{-1}$ using pUC19 as reference plasmid, no pGI2e transformants could be obtained, suggesting that pGI2 is unable to replicate in E. coli.

\section{Lagging strand replication}

In pGI2, a 250 bp sequence $62 \%$ identical to the sso of pBAA1, called $s \mathrm{O}_{t}$, could be found in the intervening sequence between ORF3 and the mob gene (Fig. 1, coordinates 7405-7654) (Seery \& Devine, 1993). Beside the structural conservation (five stem-loop structures), three conserved motifs that were identical in pBAA1 and pGI2 could also be delineated (Fig. 3). These secondary structures and conserved motifs were also identified in two RCR plasmids co-residing in B. thuringiensis israelensis, pTX14-3 (Madsen et al., 1993) and pTX141 (L. Andrup, unpublished results, EMBL accession no. U67921), and in several B. subtilis RCR plasmids (Meijer et al., 1998). In pPL1, a RCR plasmid isolated from the Gram-positive Marinococcus halophilus, a region simi- 


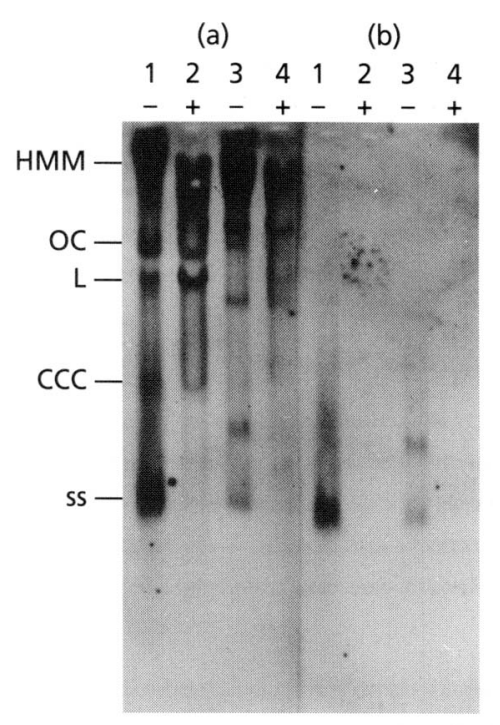

Fig. 4. Detection of pGI2 ssDNA by Southern hybridization. Total DNA from $B$. thuringiensis HD73, transformed with the appropriate plasmids, was run on an $0.8 \%(\mathrm{w} / \mathrm{v})$ agarose gel with $(+)$ or without $(-)$ prior $\$ 1$ nuclease treatment. One half of the gel was denatured before blotting (a) whereas the other half was not (b). The probe used was a DIG-labelled $1137 \mathrm{bp}$ fragment corresponding to a Kpn!-Nhel pGI2 fragment harbouring the rep gene. Lanes: 1 and 2, pGI2204c; 3 and 4, pGI2210. For the first two lanes, the positions of the singlestranded (ss), covalently closed circular (CCC), linear (L), open circular (OC) and high-molecular-mass multimer (HMM) DNA molecules are marked.

lar to the first half of the pTX14-1 sso has also been reported (Louis \& Galinski, 1997). As shown in Fig. 3, the first two conserved blocks and inverted repeat (IR) structures were present, whereas the third consensus sequence and the last three stem-loops usually found in $s \mathrm{so}_{t}$ could not be detected. For the $B$. subtilis $s \mathrm{~s}_{\mathrm{t}}$ structures, sequence similarity indicated two different subtypes, called $s s_{\mathrm{t} 1}$ (e.g. pBAA1) and $s \mathrm{ot}_{\mathrm{t} 2}$ (e.g. pTA1040) (Meijer et al., 1995). The sso from one subtype displayed $96 \%$ identity, whereas both subtypes showed $77 \%$ identity. Our alignments suggested that the sso of pGI2, pTX14-1, pTX14-3 and pPL1 cannot be considered as one of these two subtypes, nor do they belong to the same subtype as their identity does not exceed $80 \%$. Yet they all clearly fit the general characteristics typical for $s s_{\mathrm{t}}$, with the exception of pPL1, where only the first half of the structures was conserved (Fig. 3).

Although deletion experiments to delineate the active sso for pBAA1 suggested that only the first three stem-loops were important for efficient ssDNA conversion (Seery \& Devine, 1993), a similar experiment performed with pTA1060 indicated the involvement of all five stem-loops (Meijer et al., 1998). Previous experiments with pTX14-3 sso had shown that small amounts of ssDNA could be detected only when the full sso or the first and part of the second stem-loop was deleted. Moreover, rifampicin treatment of cultures harbouring pTX14-3 did not result in ssDNA accumu- lation, suggesting that the pTX14-3 sso might follow a RNA-polymerase-independent route to render ssDNA molecules double-stranded (Boe et al., 1991; Madsen et al., 1993). Consistent with these results, pBAA1 analysis revealed that most ssDNA was accumulated when the cells were harvested $10 \mathrm{~min}$ after rifampicin treatment. Longer incubation resulted in ssDNA decline (Seery \& Devine, 1993). To unambiguously prove that pGI2 is a RCR plasmid, ssDNA detection experiments were performed with the appropriate pGI2 derivatives. As in the case of pTX14-3, no pGI2 ssDNA could be detected in its host strain, B. thuringiensis H1.1, not even after rifampicin treatment (data not shown). This suggested that ssDNA conversion is extremely efficient, and might follow a RNA-polymerase-independent pathway. Southern hybridization was then performed on total DNA isolated from exponentially growing cultures of $B$. thuringiensis $\mathrm{HD} 73$, transformed with $\mathrm{pG} \mathbf{2}$ derivatives either lacking the sso (pGI2210) or harbouring only the first stem-loop (pGI2204c), which had been treated with rifampicin $10 \mathrm{~min}$ before harvesting. As can be seen in Fig. 4, both derivatives did accumulate ssDNA (lanes 1 and 3 for pGI2204c and pGI2210, respectively) eliminated by the $S 1$ treatment (lanes 2 and 4) and specifically transferred in non-denaturing conditions (Fig. 4b, lanes 1 and 3). The presence of high-molecularmass plasmid multimers is also indicative of a RCR mechanism.

\section{pGI2 mobilization module}

Upon DNA sequence analysis of pGI2, an ORF displaying similarities to those encoding 'site-specific recombinases' from other Gram-positive plasmids was found (Mahillon \& Seurinck, 1988) (labelled $m o b$ in Fig. 1 , coordinates 7829-9166). A more detailed study of these so-called mobilization proteins also revealed two conserved motifs in their N-terminal parts (Ilyina \& Koonin, 1992; Guzmán \& Espinosa, 1997). In Fig. 5, a selection of representative Mob or Mob-related proteins (including those of pTX14-1 from $B$. thuringiensis and pPL1 from $M$. halophilus) were compared using the COMPARE, BESTFIT and GAP programs. It should be noted that a -1 frameshift has been introduced at the end of the originally proposed mob gene of pTX14-3 (Andrup et al., 1991) (around nucleotide position 400). It extended the protein by 71 residues highly similar to those present in the pGI2 and pTX14-1 Mob proteins.

The relationship among these proteins is strikingly different depending on whether one considers their $\mathrm{N}$ or C-terminal half. Taking a threshold of $30 \%$ identity, these proteins displayed a subdomanial organization. Specifically, pairwise alignments of the $\mathrm{N}$ terminus suggested two groups: those from pPL1 and pTX14-1 that are similar to the pTX14-3 Mob-like protein on the one hand, and the Mob of pGI2, pMV158 and pTB53 on the other. Analysis of the $\mathrm{C}$ terminus indicated different relationships: the four most related proteins are those from pGI2, pTX14-3, pTX14-1 and, to a lesser extent, pTB53; that of pPL1 is distantly related (less than $25 \%$ identity) and that of pMV158 is apparently unrelated. 

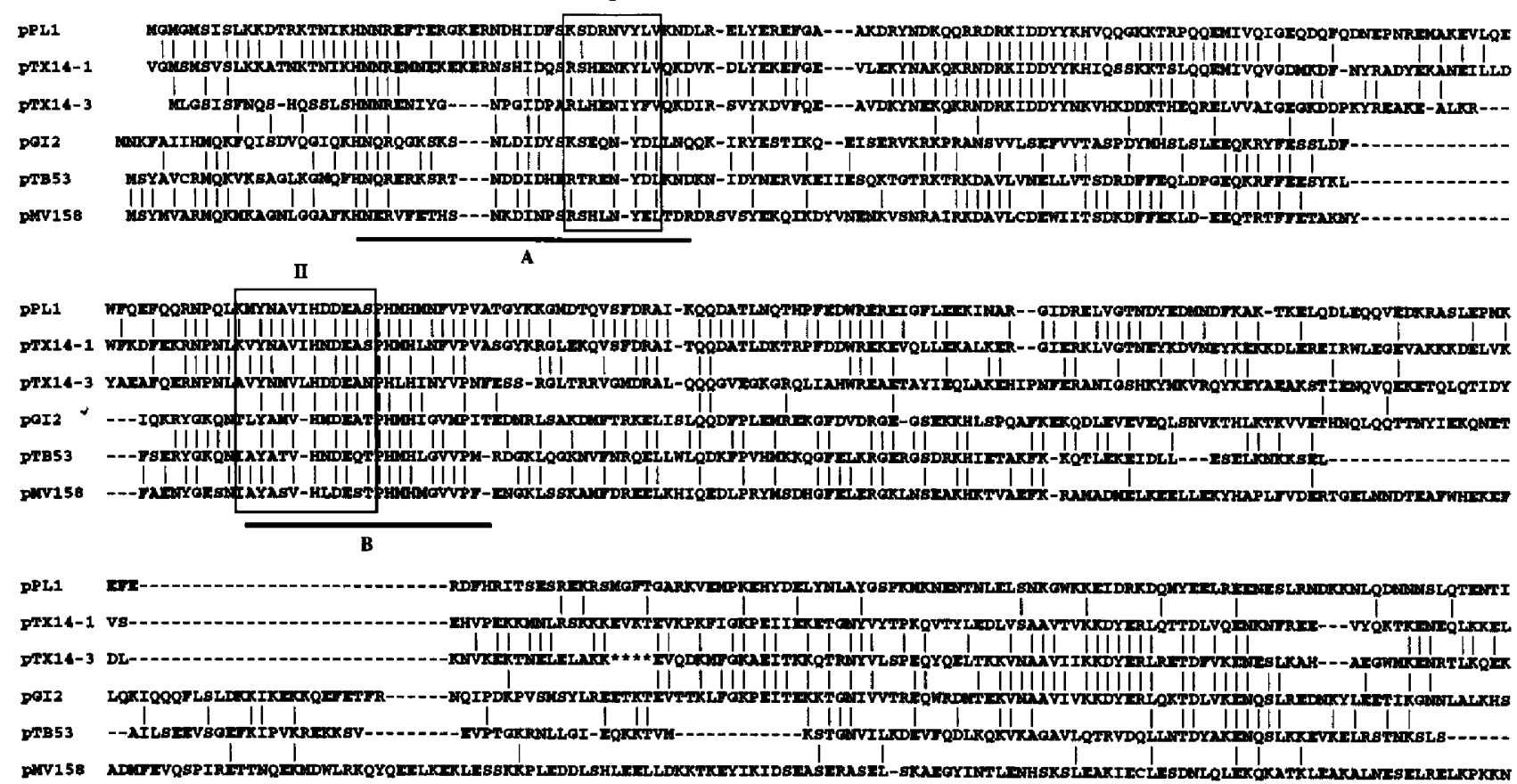

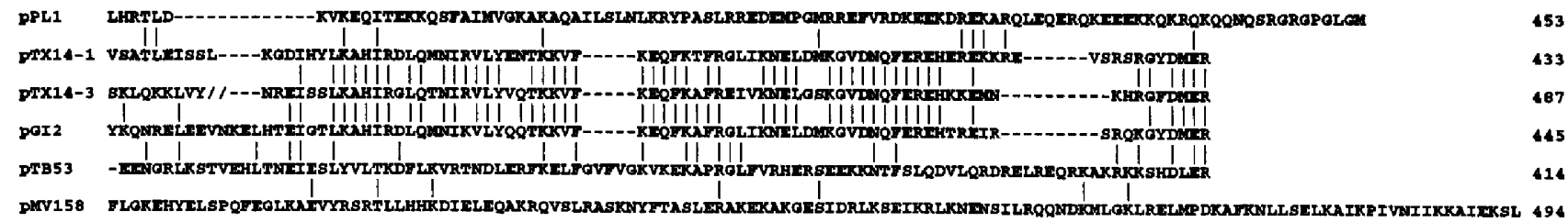

Fig. 5. Alignment of the Mob protein from pGI2 with that from pMV158, pTB53, pTX14-3, pTX14-1 and pPL1. The alignment was performed by pairwise comparison based on the findings of llyina \& Koonin (1992), Guzmán \& Espinosa (1997) and the programs BESTFIT and GAP (GCG). The conserved regions described by Guzmán \& Espinosa are underlined (A and B), whereas those described by llyina \& Koonin are boxed (I and II). In pTX14-3 Mob, the symbol **** represents a 71 amino acid region ignored in order to optimize the alignments, whereas the symbol // indicates the position of the new C-terminal end of the pTX14-3 Mob protein. This C-terminal end extends the original proposed sequence by 71 amino acids that are highly homologous to the pTX14-1 and pGI2 C-termini; it requires the introduction of a frameshift around nucleotide position 400 in PTX14-3 sequence (resulting in a frame 1 to 3 shift). The active tyrosine is that conserved within box $\mathrm{I}$. The length of each protein is also indicated.

Sequence comparisons done by Ilyina \& Koonin (1992) showed that the actual Mob proteins and those from the pTX14-3 group all possessed two conserved boxes in their N-terminal parts, although gaps had to be introduced within the conserved domains in order to match those of the pTX14-3 group (Fig. 5, boxes I and II). More recent alignments performed by Guzmán \& Espinosa (1997) with pMV158 Mob also indicated two conserved regions. In this case however, conservation with the corresponding regions in the pTX14-3 group was clearly much lower (Fig. 5, regions $\mathrm{A}$ and $\mathrm{B}$ ). In addition, the Mob-like proteins from the pTX14-3 group have a different spacing between the two boxes in comparison with those of the other group.

The corresponding structural features of these mobilization modules are the oriT sites, the cognate sequences recognized and nicked by the Mob proteins. These loci are also highly similar among different members of the same family. The putative pGI2 oriT could be located through alignment, as it is almost identical to that of pTA1015 and pTA1060 (Meijer et al., 1998); this $30 \mathrm{bp}$ sequence is located immediately downstream of the sso, $80 \mathrm{bp}$ upstream of pGI2 mob gene (Fig. 1, coordinates 7721-7750).

\section{Both pGI2 and pTX14-3 Mob-oriT modules are functional}

The mobilizing capacity of pTX14-1 and pPL1 remains untested, and the function of pTX14-3 mob has not yet been settled. Indeed, previous experiments using the aggregation-mediated conjugation system of pXO16 from $B$. thuringiensis serovar israelensis could not associate a mobilization-enhancing activity with the putative Mob protein (Andrup et al., 1995). However, this conjugation system was recently shown to mobilize many different plasmids, both natural and artificial, 
Table 3. Transfer frequency of pAW63 and pTX14-3 derivatives

Matings were between strains of $B$. thuringiensis serovar israelensis. Data are the mean number of transconjugants per recipient $(n=3)$.

\begin{tabular}{|c|c|c|}
\hline Donor & $\begin{array}{c}\text { Transfer of } \\
\text { pAW63:: Tn 5401* }\end{array}$ & $\begin{array}{c}\text { Transfer of } \\
\text { p'X14-3 derivative }+\end{array}$ \\
\hline GBJ001 (pAW63 2 Tn5401, pAND007) & $7 \cdot 7 \pm 2 \cdot 5 \times 10^{-3}$ & $2.1 \pm 0.56 \times 10^{-3}$ \\
\hline 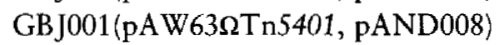 & $8 \cdot 3 \pm 2 \cdot 2 \times 10^{-3}$ & $1.3 \pm 0.44 \times 10^{-7}$ \\
\hline
\end{tabular}

* Transconjugants selected on agar plates containing nalidixic acid and tetracycline.

†Transconjugants selected on agar plates containing nalidixic acid and chloramphenicol.

Table 4. pGI2 mobilization activity

\begin{tabular}{|llc|}
\hline pGI2 derivative & \multicolumn{1}{c|}{ Description* $^{*}$} & Mobilization† \\
\hline pGI208 & Tn4430 ORF2 ORF3 sso B-44 oriT mob & $2.2 \times 10^{-4}$ \\
pGI2203 & $\Delta$ Tn4430 ORF2 ORF3 sso B-44 oriT mob & $3.2 \times 10^{-3}$ \\
pGI2205 & $\Delta$ Tn4430 ORF2 ORF3 $\Delta$ sso $\Delta \mathrm{B}-44$ oriT mob & $2.5 \times 10^{-7}$ \\
pGI2209 & $\Delta$ Tn4430 ORF2 ORF3 $\Delta s s o \Delta$ B-44 $\Delta$ oriT mob & $3.5 \times 10^{-8}$ \\
pGI2211 & $\Delta$ Tn4430 ORF2 ORF3 sso B-44 oriT $\Delta$ mob & $6.2 \times 10^{-7}$ \\
pGI2204-49 & $\Delta$ Tn4430 $\Delta$ ORF2 $\Delta$ ORF3 $\Delta s s o \Delta$ B-44 $\Delta$ oriT $\Delta$ mob & $<10^{-8}$ \\
\hline
\end{tabular}

*The structural organization of these derivatives is shown in Fig. 1. B-44 represents the $44 \mathrm{bp}$ intervening sequence between the putative sso and oriT sites of pGI2. This region is conserved among the pTX14-1, pTX14-3, pPL1 and pTB53 plasmids.

t The mobilization frequency of different pGI2 derivatives was tested in $B$. thuringiensis serovar kurstaki HD73. Data are the mean number of transconjugants per recipient $(n \geqslant 2$, except for pGI220449 , where $n=1$ ).

including those lacking mob genes and oriT sites (Andrup et al., 1996). It is thus fundamentally different from other known transfer systems and is inappropriate to study the potential mobilization activity of pTX14-3. Therefore, the newly described pAW63 conjugation system of $B$. thuringiensis serovar kurstaki HD73 was used. This system can indeed mobilize non-conjugative plasmids via a Mob-dependent mechanism (Wilcks et al., 1998).

The conjugation experiments were performed in a $B$. thuringiensis serovar israelensis strain cured of all its plasmids. The pAW63 conjugative plasmid, tagged with the Tn5401 transposon (Wilcks et al., 1998), was used to transfer the pAND007 $\left(\mathrm{Mob}^{+}\right)$and pAND008 $\left(\mathrm{Mob}^{-}\right)$ derivatives of pTX14-3 (Andrup et al., 1995). As shown in Table 3, inactivation of the $m o b$ gene (pAND008) had a drastic effect on the transfer frequency of pTX14-3, with more than a $10^{4}$-fold reduction, whilst pAW63 transfer frequency remained similar in both experiments. These results confirmed a contribution of Mob to pTX14-3 plasmid mobility.

Similar experiments were performed with several pGI2 derivatives. In the case of an intact Mob-oriT module (Table 4, pGI2203), 3.2 $\times 10^{-3}$ transconjugants were obtained per recipient cell, a frequency similar to that obtained with the corresponding pTX14-3 derivative (Table 3, pAND007). Likewise, inactivation of the $m o b$ gene (pGI2211) resulted in a $5 \times 10^{3}$-fold decrease in the transfer frequency, a situation analogous to that of pAND008. Removal of the oriT site (pGI2209) reduced conjugal transfer severely, whereas removal of both elements (pGI2204-49) abolished it totally. It is interesting to note that although pGI2205 harboured both the putative oriT and mob gene, its mobilization activity is essentially similar to that of the $\mathrm{Mob}^{-}$ derivative (pGI2211). This observation suggests the possible involvement of the $44 \mathrm{bp}$ intervening region located between the $m o b$ gene and the $s \mathrm{O}_{\mathrm{t}}$-structure in the transfer activity. This region is also present in pTX14-3, pTX14-1 and pPL1 (Fig. 3), and a similar sequence was also detected upstream of the pTB53 mob gene (EMBL accession no. D14852). Finally, a 10-fold reduction in mobilization efficiency was observed between pGI2203 and pGI208. Although no simple explanation can account for this observation, it could possibly result from the size difference between the two derivatives and/or interference of $\operatorname{Tn} 4430$ in the transfer process.

\section{Segregational stability}

In order to assess the impact of the sso and Mob-oriT modules on pGI2 segregational stability, four derivatives (pGI2203, pGI2211, pGI2209 and pGI2205, Fig. 1) were transformed into strain AW17 of B. thuringiensis HD73 
Table 5. Segregational stability of $\mathrm{pGI} 2$ derivatives in B. thuringiensis HD73

Plasmid stability was estimated as the percentage of B. thuringiensis transformants retaining their respective pGI2 derivatives $\left(\mathrm{Ery}^{\mathrm{R}} / \mathrm{Str}^{\mathrm{R}}\right)$ after $10-40$ generations at $30^{\circ} \mathrm{C}$ without antibiotic selection. The features and structural organization of the pGI2 derivatives are shown in Table 4 and Fig. 1, respectively,

\begin{tabular}{|lrrrr|}
\hline pGI2 derivative & \multicolumn{4}{c|}{ No. of generations : } \\
\cline { 2 - 5 } & $\mathbf{1 0}$ & $\mathbf{2 0}$ & $\mathbf{3 0}$ & $\mathbf{4 0}$ \\
\hline pGI2203 & 100 & 100 & 100 & 100 \\
pGI2211 & 100 & 99 & 98 & 95 \\
pGI2209 & 100 & 81 & 45 & 24 \\
pGI2205 & 95 & 86 & 77 & 59 \\
\hline
\end{tabular}

and monitored for stability during 40 generations. As shown in Table 5, inactivation of Mob (pGI2211) had little effect on plasmid stability, even after 40 generations (only $5 \%$ of cured strains). This contrasted with the drop in plasmid-bearing cells observed when the sso site was deleted (pGI2205). Removal of both sso and oriT (pGI2209) resulted in an even more deleterious effect, with only $24 \%$ transformants left after 40 generations. These observations indicated that the pGI2 sso, identified by its similarity to other sso, is not indispensable but substantially contributes to plasmid stability, presumably by avoiding accumulation of ssDNA. They also suggested a possible interference of pGI 2 oriT in this process, although at this stage one cannot discriminate between a direct or indirect effect.

\section{pGI2 distribution in the $B$. cereus group}

Distribution of the pGI2 replicon among bacteria of the $B$. cereus group was examined by amplifying a $530 \mathrm{bp}$ region located within the rep gene from 34 strains of $B$. thuringiensis, 10 of $B$. cereus and 13 of Bacillus mycoides (Table 1). Whereas 10/34 B. thuringiensis strains contained a PCR product with the expected $0.5 \mathrm{~kb}$ size $(B$. thuringiensis Bt1, HD14, T01 002, T01 027, T03B001, T04B001, T16 001, T18 001, T21 001, T42 001), none of the B. cereus and B. mycoides strains gave any PCR signal. Interestingly, in all the strains examined but one (HD14), pGI2 was only found in those also containing the $\mathrm{pGI} 3$ replicon (Hoflack et al., 1997), the largest of the three cryptic plasmids from $B$. thuringiensis H1.1 (Mahillon et al., 1988).

\section{DISCUSSION}

Southern hybridization proved that pGI 2 is a RCR plasmid and, although its replication protein is distantly related to that of pTX14-3 ( $30 \%$ identity), pGI 2 can be considered as the second known member of the pTX143 RCR family. Moreover, deletion experiments also indicated that a 320 bp sequence located downstream of the Rep protein is indispensable for pGI2 replication. Interestingly, a very similar sequence occupies the same relative position in $\mathrm{pTX} 14-3$. It is therefore tempting to speculate that this sequence is the actual $d s o$ of both plasmids. However, such a location downstream of Rep has not been reported previously and therefore this assumption requires further confirmation.

The pGI2 sso is of the sso $_{\mathrm{t}}$-type, which is commonly found in B. subtilis [pTA1015, pTA1020, pTA1030, pTA1040, pTA1050 and pTA1060 (Meijer et al., 1995), and pBAA1 (Seery \& Devine, 1993)], and is also present in pTX14-3 (Andrup et al., 1994) and pTX14-1. sso is thought to consist of three conserved boxes and five stem-loop structures (Meijer et al., 1995). Recently, a RCR plasmid isolated from $M$. halophilus was also found to harbour an $s \mathrm{so}_{\mathrm{t}}$-like structure bearing only the first two conserved boxes and two stem-loops. Since it has been elucidated for B. subtilis that at least the first three IR are important for efficient conversion of the ssDNA intermediate (Seery \& Devine, 1993), it would be interesting to find out whether the differences between the Bacillus sso $_{\mathrm{t}}$ and the sequence found in the $M$. balophilus plasmid merely reflect host-range diversity, or imply that this pPL1 structure has no sso function.

In pGI2, ssDNA could only be detected in derivatives lacking the full sso, or harbouring only the first stem-loop. In its original B. thuringiensis H1.1 strain, no pGI2 ssDNA was observed, not even after rifampicin treatment. This implies that ssDNA conversion is very efficient and able to follow an RNA-polymerase-independent pathway. This feature had already been found for pTX14-3 sso $\mathrm{s}_{\mathrm{t}}$, where rifampicin treatment did not result in any ssDNA accumulation. Tiny amounts of ssDNA could only be observed in plasmids lacking either the full sso, or a region comprising the first stem-loop and part of the second hairpin (Boe et al., 1991). Also, ssDNA detection from cells harbouring pBAA1 showed that incubations longer than $10 \mathrm{~min}$ with rifampicin resulted in ssDNA decline, arguing for the existence of a RNA-polymerase-independent pathway in addition to the rifampicin-sensitive process. For another sso type, namely $s O_{\mathrm{w}}$ from $\mathrm{pWV01,} \mathrm{the}$ RNA-independent pathway was studied in detail (Seegers et al., 1995). The results indicated that the first of two stem-loops was sufficient for ssDNA conversion using the alternative route, whilst the RNA-dependent route needed both IR (Seegers et al., 1995). This is probably not the case for sso $_{\mathrm{t}}$ from $\mathrm{pGI} 2$, since the construct harbouring the first stem-loop accumulated significant amounts of ssDNA.

Another striking result is that obtained with the pGI2 mob module that proved to be active in mobilizing pGI2 during transfer mediated by pAW63 from $B$. thuringiensis serovar kurstaki HD73. Some doubts had arisen because, although the pGI2 Mob N-terminal half showed homology to the mobilization protein of pMV158, its C terminus was related to the Mob-like protein from pTX14-3 shown to be active in plasmid 
segregational stability rather than mobilization (Andrup et al., 1995). It should be noted however that the pTX143 mobilization experiments were conducted with pXO16 from $B$. thuringiensis serovar israelensis, whose conjugation apparatus is distinct from that of pAW63 (Andrup et al., 1995, 1996). Experiments presented here solved this ambiguity by demonstrating that pTX14-3, like $\mathrm{pGI} 2$, bore a bona fide Mob protein able to use the pAW63 conjugation apparatus for mobilization.

Detailed sequence comparison of the Mob-like proteins of pGI2 and pTX14-3 with related proteins identified a bidomanial organization (Fig. 5). Considering their Nterminal segment, two groups could be distinguished: that of pGI2, pTB53 and pMV158, and another consisting of the Mob proteins from pTX14-3, pTX14-1 and pPL1. Although both groups seemed to possess the two conserved motifs commonly present in the $\mathrm{N}$ terminus of active Mob proteins, including the active tyrosine residue (llyina \& Koonin, 1992; Guzmán \& Espinosa, 1997), gaps were required to align the conserved sequences with their counterparts in the pTX143 group. Could at least some of these subdomanial relationships be correlated with other shared features? At least two observations can be made. Whereas pGI2, pTB53 and pMV158 do have a related oriT, no similar site could be detected in pPL1, pTX14-1 and pTX14-3 (data not shown). A second, although less striking, association can be found between the conserved $44 \mathrm{bp}$ DNA sequence (adjacent to the sso) and the related Cterminal part of the Mob proteins from pGI2, pTX14-1, pTX14-3, pTB53 and possibly pPL1. The possible function of this association remains however unclear.

The recently sequenced $60 \mathrm{~kb}$ pMRC01 plasmid from $L$. lactis subsp. lactis contained at least 64 putative ORFs organized into three functional domains, those of plasmid transfer, plasmid replication and bacteriocin production (Dougherty et al., 1998). In the latter, the last 286 aa ORF43 displayed $39 \%$ identity with pGI2 ORF2. Unfortunately, no function has yet been assigned to this putative protein, only its location downstream of the lacticin operon. Thus, with the exception of ORF2 and ORF3 to which no putative function can yet be assigned, the $9672 \mathrm{bp}$ pGI2 plasmid appears as an intriguing reservoir of various transfer and rearrangement modules articulated on transpositional and site-specific recombination (Tn4430, Mahillon \& Lereclus, 1988) or ssDNA displacement and transfer activities (Rep and Mob). This clustering of DNA-redesigning-associated functions into a distinct genomic entity is probably providing the bacteria with the necessary assets to cope with the uncertainties of their environment without having to challenge the stability of their chromosome.

\section{ACKNOWLEDGEMENTS}

We wish to thank M. Van Montagu and M. Peferoen in whose laboratories part of this work was performed, and $K$. van Audenhove for providing us with pGI208, pGI2203 and pGI2204. This work was supported by grants from the IW'T (Vlaams Instituut voor de bevordering van het
Wetenschappelijk en Technologisch Onderzoek in de Industrie) and FVRS (ronds National de la Recherche Scientificue) from which .]. Mahillon is a Research Associate. A. Wilcks was supported by the Danish Natural Science Rescarch Council.

\section{REFERENCES}

Andrup, L., Bolander, G., Boe, L., Madsen, 5. M., Nielsen, T. T. \& Wasserman, K. (1991). Identification of a gene (mob 4-3) encoding a mobilization protein from the Bacillus thuringiensis subsp. israelensis plasmid pTX14-3. Nucleic Acids Res 19, 2780.

Andrup, L., Damgaard, J., Wassermann, K., Boe, L., Madsen, S. M. \& Hansen, F. G. (1994). Complete nucleotide sequence of the Bacillus thuringipnsis suhsp. israelensis plasmid $\mathrm{PTX} 14-3$ and its correlation with biological properties. Plasmid 31, 7288 .

Andrup, L., Bendixen, H. H. \& Jensen, G. (1995). Mobilization of Bacillus thuringiensis plasmid $\mathrm{TTX} 14-3$. Plasmid 33, 159-167.

Andrup, L., Jorgensen, O., Wilcks, A., Smidt, L. \& Jensen, G. B. (1996). Mobilization of 'nonmobilizatle' plasmids by the aggregation mediared conjugation system of Bacillus thuringiensis. Plastidid 36, 75-85.

Bell, J. A. \& Friedman, S. B. (1994). Genetic struccure and diversity within local populations of Bacillus mycoides. Evolution 48, 1698-1714.

Boe, L., Gros, M. F., te Riele, H., Ehrlich, S. D. \& Gruss, A. (1989). Replication origins of single-stranded-DNA plasmid pUB110. I Bacteriol 171, 3366-3372.

Boe, L., Nielsen, T. T., Madsen, S. M., Andrup, L. \& Bolander, G. (1991). Cloning and characterization of two plasmids from Bacillus thuringiensis in Bacillus subtilis. Plasmid 25, 190-197.

Carlson, R., Caugant, D. A. \& Kolstø, A.-B. (1994). Genotypic diversity among Bacillus cereus and Bacillus thuringiensis strains. Appl Environ Microbiol 60, 1719-1725.

Devine, K. M., Hogan, S. T., Higgins, D. G. \& McConnel, D. J. (1989). Replication and segregational stability of Bacillus plasmid pBAA1. I Bacleriol 171, 1166-1172.

Dougherty, B. A., Hill, C., Weidman, J. F., Richardson, D. R., Venter, J. C. \& Ross, R. P. (1998). Sequence and analysis of the $60 \mathrm{~kb}$ conjugative, bacteriocin-producing plasmid pMRCOl from Lactococus lactis DPC 3147. Mol Microbiol 29, 1029-1038.

Espinosa, M., del Solar, G., Rojo, F. \& Alonso, J. C. (1995). Plasmid rolling circle replication and its control. PEMS Microbiol Lett $130,111-120$.

Guzmán, L. M. \& Espinosa, M. (1997). The mobilization protein, MobM, of the streptococed plasmid pMV 158 specifically cleaves supercoiled DNA at the plasmid oriT. I Mol Biol 266, 688-702.

Hoflack, L., Seurinck, J. \& Mahillon, J. (1997). Nucleotide sequence and characterization of the cryptic Bacillus thuringiensis plasmid pGI3 reveal a new family of roiling circle replicons. J Bacteriol $197,5000-5008$.

Ilyina, T. V. \& Koonin, E. V. (1992). Conserved sequence motifs in the initiator proteins for rolling circle DNA replication encoded by diverse replicons from euhacteria, eucaryotes and archaebacteria. Nucleic Acids Res 20, 3279-3285.

Jannière, L., Gruss, A. \& Ehrlich, D. (1993). Plasmids. In Bacillus subtilis and Other Gram-positive Bacteria: Biochemistry, Pbysiology, and Molecular Genetics, pp. 625-644. Edited by A. L. Sonenshein, J. A. Hoch \& R. Losick. Washington, DC: American Society for Microbiology.

Jensen, G. B., Wilcks, A., Petersen, S. S., Damgaard, J., Baum, J. A. \& Andrup, L. (1995). The genetic basis of the aggregation system 
in Bacillus thuringiensis subsp. israelensis is located on the large conjugative plasmid pX016. J Bacteriol 177, 2914-2917.

Jensen, G. B., Andrup, L., Wilcks, A., Smidt, L. \& Poulsen, O. M. (1996). The aggregation-mediated conjugation system of Bacillus thuringiensis subsp. israelensis: host range and kinetics of transfer. Curr Microbiol 33, 228-236.

Jones, D. \& Errington, J. (1987). Construction of improved bacteriophage $\phi 105$ vectors for cloning by transfection in Bacillus subtilis. J Gen Microbiol 133, 483-492.

Josson, K., Scheirlinck, T., Michiels, F., Platteeuw, C., Stanssens, P., Joos, H., Dhaese, P., Zabeau, M. \& Mahillon, J. (1989). Characterization of a Gram-positive broad-host-range plasmid isolated from Lactobacillus hilgardii. Plasmid 21, 9-20.

Khan, S. A. (1997). Rolling-circle replication of bacterial plasmids. Microbiol Mol Biol Rev 61, 442-455.

Khan, S. A. \& Novick, R. (1982). Structural analysis of plasmid pSN2 in Staphylococcus aureus: no involvement in enterotoxin B production. J Bacteriol 149, 642-649.

Lanka, E. \& Wilkins, B. (1995). DNA processing reactions in bacterial conjugation. Annu Rev Biochem 64, 141-169.

Léonard, C., Chen, Y. \& Mahillon, J. (1997). Diversity and differential distribution of IS231, IS232 and IS240 among Bacillus cereus, Bacillus thuringiensis and Bacillus mycoides. Microbiology 143, 2537-2547.

Lereclus, D. \& Arantes, O. (1992). $s p b A$ locus ensures the segregational stability of pHT1030, a novel type of Gram-positive replicon. Mol Microbiol 6, 35-46.

Louis, P. \& Galinski, E. A. (1997). Identification of plasmids in the genus Marinococcus and complete nucleotide sequence of plasmid pPL1 from Marinococcus halophilus. Plasmid 38, 107-114.

Madsen, S. M., Andrup, L. \& Boe, L. (1993). Fine mapping and DNA sequence of replication functions of Bacillus thuringiensis plasmid pTX14-3. Plasmid 30, 119-130.

Mahillon, J. \& Lereclus, D. (1988). Structural and functional analysis of $\operatorname{Tn} 4430$ : identification of an integrase-like protein involved in the co-integrate-resolution process. EMBO $\mathrm{J} \mathrm{7}$, 1515-1526.

Mahillon, J. \& Seurinck, J. (1988). Complete nucleotide sequence of pGI2, a Bacillus thuringiensis plasmid containing Tn4430. Nucleic Acids Res 16, 11827-11828.

Mahillon, J., Hespel, F., Pierssens, F. \& Delcour, J. (1988). Cloning and partial characterization of three small cryptic plasmids from Bacillus thuringiensis. Plasmid 19, 169-173.

Mahillon, J., Rezsobhazy, R., Hallet, B. \& Delcour, J. (1994). IS231 and other Bacillus thuringiensis transposable elements: a review. Genetica 93, 13-26.

Meijer, W. J. J., de Jong, A., Wisman, G. B. A., Tjalsma, H., Venema, G. \& Bron, S. (1995). The endogenous Bacillus subtilis (natto) plasmids pTA1015 and pTA1040 contain signal peptidaseencoding genes: identification of a new structural module on cryptic plasmids. Mol Microbiol 17, 621-631.

Meijer, W. J. J., Wisman, G. B. A., Terpstra, P., Thorsted, P. D., Thomas, C. M., Holsappel, S., Venema, G. \& Bron, S. (1998).
Rolling circle plasmids from Bacillus subtilis : complete nucleotide sequences and analyses of genes of pTA1015, pTA1040, pTA1050 and pTA1060, and comparison with related plasmids from Grampositive bacteria. FEMS Microbiol Rev 21, 337-368.

Nakamura, L. K. \& Jackson, M. A. (1995). Clarification of the taxonomy of Bacillus mycoides. Int J Syst Bacteriol 45, 46-49.

Narumi, I., Nakayama, I., Nakamoto, S. \& Kihara, H. (1995). Bacillus stearothermopbilus plasmid pSTK1 replicon is functional in Escherichia coli. Biotechnol Lett 17, 475-480.

Noirot-Gros, M. F., Bidnenko, V. \& Ehrlich, S. D. (1994). Active site of the replication protein of the rolling circle plasmid pC194. EMBO J 13, 4412-4420.

Novick, R. P. (1989). Staphylococcal plasmids and their replication. Annu Rev Microbiol 45, 537-565.

Sambrook, J., Fritsch, E. F. \& Maniatis, T. (1989). Molecular Cloning: a Laboratory Manual, 2nd edn. Cold Spring Harbor, NY: Cold Spring Harbor Laboratory.

Seegers, J. F. M. L., Zhao, A. C., Meijer, W. J. J., Khan, S. A., Venema, G. \& Bron, S. (1995). Structural and functional analysis of the single-strand origin of replication from the lactococcal plasmid pWV01. Mol Gen Genet 249, 43-50.

Seery, L. \& Devine, K. M. (1993). Analysis of features contributing to activity of the single-stranded origin of Bacillus plasmid pBAA1. J Bacteriol 175, 1988-1994.

Seery, L. T., Nolan, N. C., Sharp, P. M. \& Devine, K. M. (1993). Comparative analysis of the pC194 group of rolling circle plasmids. Plasmid 30, 185-196.

del Solar, G., Kramer, M. G., Ballester, S. \& Espinosa, M. (1993). Replication of the promiscuous plasmid pLS1: a region encompassing the minus origin of replication is associated with stable plasmid inheritance. Mol Gen Genet 241, 97-105.

Voskuil, M. I. \& Chambliss, G. H. (1993). Rapid isolation and sequencing of purified plasmid DNA from Bacillus subtilis. Appl Environ Microbiol 59, 1138-1142.

Wang, P.-Z., Projan, S. J., Henriquez, V. \& Novick, R. (1993). Origin recognition specificity in pT181 plasmids is determined by a functionally asymmetric palindromic DNA element. $E M B O J$ $12,45-52$.

Wertman, K. F., Wyman, A. F. \& Botsein, D. (1986). Host/vector interactions which affect the viability of recombinant phage lambda clones. Gene 49, 253-262.

Wilcks, A., Jayaswal, N., Lereclus, D. \& Andrup, L. (1998). Characterization of plasmid pAW63, a second self-transmissible plasmid in Bacillus thuringiensis subspecies kurstaki HD73. Microbiology 144, 1263-1270.

Woodcock, D. M., Crowther, P. J., Doherty, J., Jefferson, S., DeCruz, E., Noyer-Weidner, M., Smith, S. S., Michael, M. Z. \& Graham, M. W. (1989). Quantitative evaluation of Escherichia coli host strains for tolerance to cytosine methylation in plasmid and phage recombinants. Nucleic Acids Res 17, 3469-3478.

Received 11 January 1999; revised 8 March 1999; accepted 16 March 1999. 